Acta Crystallographica Section E

Structure Reports

Online

ISSN 1600-5368

\section{3-(4-Bromophenylsulfinyl)-2,5-dimethyl- 1-benzofuran}

\section{Hong Dae Choi, ${ }^{\text {a }}$ Pil Ja Seo ${ }^{a}$ and Uk Lee ${ }^{b *}$}

a Department of Chemistry, Dongeui University, San 24 Kaya-dong Busanjin-gu, Busan 614-714, Republic of Korea, and ${ }^{\mathbf{b}}$ Department of Chemistry, Pukyong National University, 599-1 Daeyeon 3-dong Nam-gu, Busan 608-737, Republic of Korea

Correspondence e-mail: uklee@pknu.ac.kr

Received 6 January 2012; accepted 21 January 2012

Key indicators: single-crystal X-ray study; $T=173 \mathrm{~K}$; mean $\sigma(\mathrm{C}-\mathrm{C})=0.003 \AA$. $R$ factor $=0.033 ; w R$ factor $=0.087$; data-to-parameter ratio $=19.2$.

In the title compound, $\mathrm{C}_{16} \mathrm{H}_{13} \mathrm{BrO}_{2} \mathrm{~S}$, the 4-bromophenyl ring makes a dihedral angle of $87.87(6)^{\circ}$ with the mean plane of the benzofuran fragment. In the crystal, molecules are linked by a weak $\pi-\pi$ interaction between the 4-bromophenyl rings [centroid-to-centroid distance $=3.907$ (3) $\AA$, interplanar distance $=3.528$ (3) $\AA$ and slippage $=1.679(3) \AA$ ] .

\section{Related literature}

For the pharmacological activity of benzofuran compounds, see: Aslam et al. (2009); Galal et al. (2009); Khan et al. (2005). For natural products with benzofuran rings, see: Akgul \& Anil (2003); Soekamto et al. (2003). For the crystal structures of related compounds, see: Choi et al. $(2010 a, b)$.

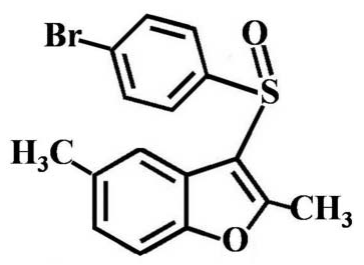

Data collection

Bruker SMART APEXII CCD

diffractometer

Absorption correction: multi-scan (SADABS; Bruker, 2009)

$T_{\min }=0.518, T_{\max }=0.746$

Refinement

$R\left[F^{2}>2 \sigma\left(F^{2}\right)\right]=0.033$

$w R\left(F^{2}\right)=0.087$

$S=1.04$

3507 reflections

12848 measured reflections 3507 independent reflections 3048 reflections with $I>2 \sigma(I)$ $R_{\text {int }}=0.044$

Data collection: APEX2 (Bruker, 2009); cell refinement: SAINT (Bruker, 2009); data reduction: $S A I N T$; program(s) used to solve structure: SHELXS97 (Sheldrick, 2008); program(s) used to refine structure: SHELXL97 (Sheldrick, 2008); molecular graphics: ORTEP-3 (Farrugia, 1997) and DIAMOND (Brandenburg, 1998); software used to prepare material for publication: SHELXL97.

This work was supported by the Blue-Bio Industry Regional Innovation Centre (grant No. RIC08-06-07) at Dongeui University as an RIC programme under the Ministry of Knowledge Economy and Busan city.

Supplementary data and figures for this paper are available from the IUCr electronic archives (Reference: KP2381).

\section{References}

Akgul, Y. Y. \& Anil, H. (2003). Phytochemistry, 63, 939-943.

Aslam, S. N., Stevenson, P. C., Kokubun, T. \& Hall, D. R. (2009). Microbiol. Res. 164, 191-195.

Brandenburg, K. (1998). DIAMOND. Crystal Impact GbR, Bonn, Germany. Bruker (2009). APEX2. SADABS and SAINT. Bruker AXS Inc., Madison, Wisconsin, USA.

Choi, H. D., Seo, P. J., Son, B. W. \& Lee, U. (2010a). Acta Cryst. E66, o543.

Choi, H. D., Seo, P. J., Son, B. W. \& Lee, U. (2010b). Acta Cryst. E66, o2551.

Farrugia, L. J. (1997). J. Appl. Cryst. 30, 565.

Galal, S. A., Abd El-All, A. S., Abdallah, M. M. \& El-Diwani, H. I. (2009). Bioorg. Med. Chem. Lett. 19, 2420-2428.

Khan, M. W., Alam, M. J., Rashid, M. A. \& Chowdhury, R. (2005). Bioorg. Med. Chem. 13, 4796-4805.

Sheldrick, G. M. (2008). Acta Cryst. A64, 112-122.

Soekamto, N. H., Achmad, S. A., Ghisalberti, E. L., Hakim, E. H. \& Syah, Y. M. (2003). Phytochemistry, 64, 831-834.

\title{
Experimental
}

\section{Crystal data}

$\mathrm{C}_{16} \mathrm{H}_{13} \mathrm{BrO}_{2} \mathrm{~S}$

$M_{r}=349.23$

Triclinic, $P \overline{1}$

$a=6.4145(3) \AA$

$b=10.0266(5) \AA$

$c=11.7639$ (6) $\AA$

$\alpha=101.606(3)^{\circ}$

$\beta=92.240(2)^{\circ}$

$$
\begin{aligned}
& \gamma=103.932(2)^{\circ} \\
& V=716.28(6) \AA^{3} \\
& Z=2 \\
& \text { Mo } K \alpha \text { radiation } \\
& \mu=3.01 \mathrm{~mm}^{-1} \\
& T=173 \mathrm{~K} \\
& 0.30 \times 0.23 \times 0.20 \mathrm{~mm}
\end{aligned}
$$




\section{supporting information}

Acta Cryst. (2012). E68, o584 [doi:10.1107/S1600536812002656]

\section{3-(4-Bromophenylsulfinyl)-2,5-dimethyl-1-benzofuran}

\section{Hong Dae Choi, Pil Ja Seo and Uk Lee}

\section{S1. Comment}

Many compounds involving a benzofuran ring have drawn much attention owing to their valuable biological properties such as antibacterial, antifungal, antitumor, and antiviral activities (Aslam et al., 2009; Galal et al., 2009; Khan et al., 2005). These benzofuran derivatives occur in a wide range of natural products (Akgul \& Anil, 2003; Soekamto et al., 2003). As a part of our continuing study of 2,5-dimethyl-1-benzofuran derivatives containing either 3-(4-fluorophenylsulfinyl) (Choi et al., 2010a) or 3-(4-chlorophenylsufinyl) (Choi et al., 2010b) substituents, we report herein the crystal structure of the title compound.

In the title molecule (Fig. 1), the benzofuran unit is planar with the mean deviation of 0.006 (2) $\AA$ from the least-squares plane defined by the nine constituent atoms. The dihedral angle between the 4-bromophenyl ring and the mean plane of the benzofuran fragment is $87.87(6)^{\circ}$. The crystal packing (Fig. 2) is further stabilised by a weak $\pi-\pi$ interaction between the 4-bromophenyl rings of adjacent molecules, with a $\mathrm{Cg} \cdots \mathrm{Cg}^{\mathrm{i}}$ distance of 3.907 (3) $\AA$ and an interplanar distance of 3.528 (3) $\AA$ resulting in a slippage of 1.679 (3) $\AA$ (Cg is the centroid of the C11-C16 4-bromophenyl ring).

\section{S2. Experimental}

3-Chloroperoxybenzoic acid 77\% (269 mg, $1.2 \mathrm{mmol}$ ) was added in small portions to a stirred solution of 3-(4-bromophenylsulfanyl)-2,5-dimethyl-1-benzofuran $(366 \mathrm{mg}, 1.1 \mathrm{mmol})$ in dichloromethane $(40 \mathrm{ml})$ at $273 \mathrm{~K}$. After being stirred at room temperature for $5 \mathrm{~h}$, the mixture was washed with saturated sodium bicarbonate solution and the organic layer was separated, dried over magnesium sulfate, filtered and concentrated at reduced pressure. The residue was purified by column chromatography (hexane-ethyl acetate, $2: 1 \mathrm{v} / \mathrm{v}$ ) to afford the title compound as a colourless solid [yield $72 \%$, m.p. $434-435 \mathrm{~K} ; R_{\mathrm{f}}=0.49$ (hexane-ethyl acetate, $2: 1 \mathrm{v} / \mathrm{v}$ )]. Single crystals suitable for X-ray diffraction were prepared by slow evaporation of a solution of the title compound in ethyl acetate at room temperature.

\section{S3. Refinement}

All $\mathrm{H}$ atoms were positioned geometrically and refined using a riding model, with $\mathrm{C}-\mathrm{H}=0.95 \AA$ for aryl and $0.98 \AA$ for methyl $\mathrm{H}$ atoms. $U_{\text {iso }}(\mathrm{H})=1.2 U_{\text {eq }}(\mathrm{C})$ for aryl and $1.5 U_{\text {eq }}(\mathrm{C})$ for methyl $\mathrm{H}$ atoms. 


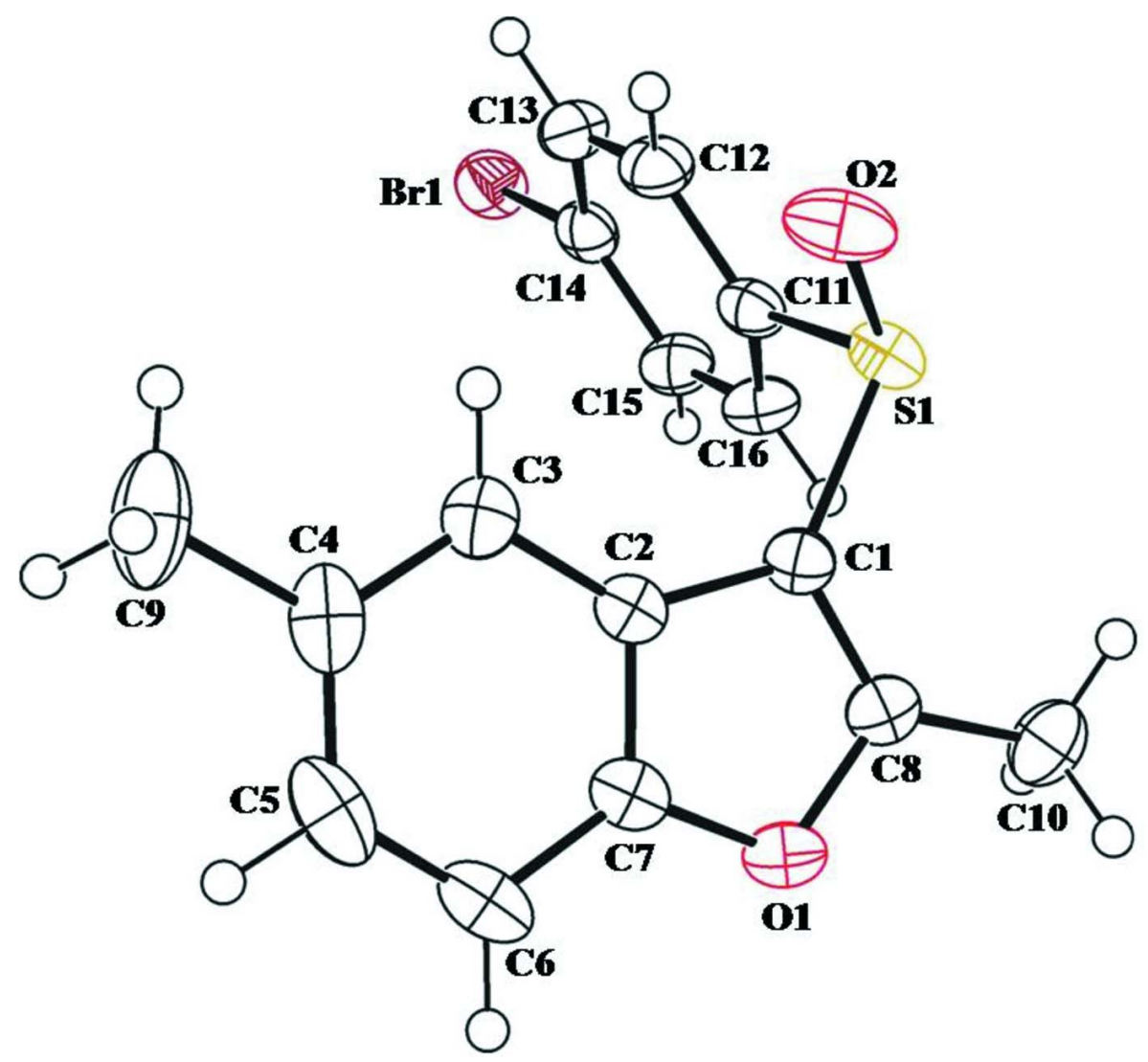

\section{Figure 1}

The molecular structure of the title compound with the atom numbering scheme. Displacement ellipsoids are drawn at the $50 \%$ probability level. $\mathrm{H}$ atoms are presented as small spheres of arbitrary radius.

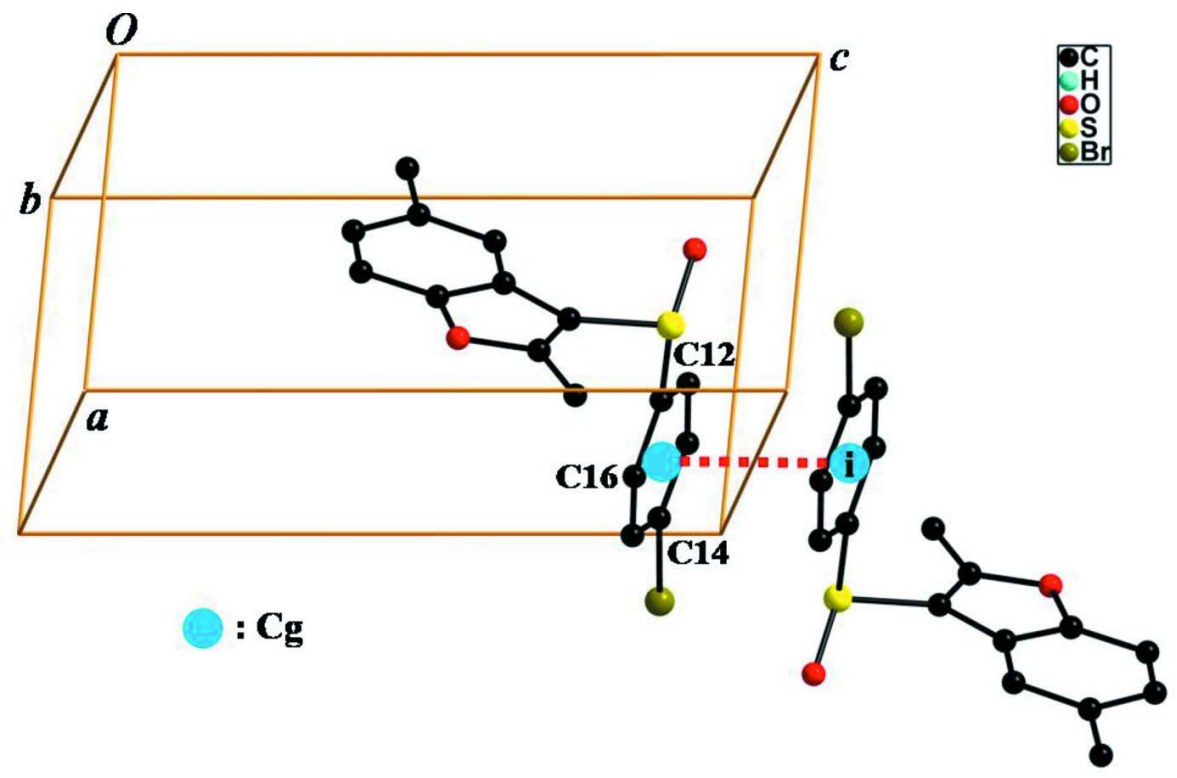

Figure 2

A view of the $\pi-\pi$ interactions (dotted lines) in the crystal structure of the title compound. All $\mathrm{H}$ atoms were omitted for clarity. [Symmetry codes: (i) $-x+2,-y+1,-z+2]$. 


\section{3-(4-Bromophenylsulfinyl)-2,5-dimethyl-1-benzofuran}

Crystal data

$\mathrm{C}_{16} \mathrm{H}_{13} \mathrm{BrO}_{2} \mathrm{~S}$

$M_{r}=349.23$

Triclinic, $P \overline{1}$

Hall symbol: -P 1

$a=6.4145(3) \AA$

$b=10.0266(5) \AA$

$c=11.7639(6) \AA$

$\alpha=101.606(3)^{\circ}$

$\beta=92.240(2)^{\circ}$

$\gamma=103.932(2)^{\circ}$

$V=716.28(6) \AA^{3}$

Data collection

Bruker SMART APEXII CCD diffractometer

Radiation source: rotating anode

Graphite multilayer monochromator

Detector resolution: 10.0 pixels $\mathrm{mm}^{-1}$

$\varphi$ and $\omega$ scans

Absorption correction: multi-scan

(SADABS; Bruker, 2009)

$T_{\min }=0.518, T_{\max }=0.746$

Refinement

Refinement on $F^{2}$

Least-squares matrix: full

$R\left[F^{2}>2 \sigma\left(F^{2}\right)\right]=0.033$

$w R\left(F^{2}\right)=0.087$

$S=1.04$

3507 reflections

183 parameters

0 restraints

Primary atom site location: structure-invariant direct methods
$Z=2$

$F(000)=352$

$D_{\mathrm{x}}=1.619 \mathrm{Mg} \mathrm{m}^{-3}$

Mo $K \alpha$ radiation, $\lambda=0.71073 \AA$

Cell parameters from 7068 reflections

$\theta=2.5-28.2^{\circ}$

$\mu=3.01 \mathrm{~mm}^{-1}$

$T=173 \mathrm{~K}$

Block, colourless

$0.30 \times 0.23 \times 0.20 \mathrm{~mm}$

12848 measured reflections

3507 independent reflections

3048 reflections with $I>2 \sigma(I)$

$R_{\text {int }}=0.044$

$\theta_{\max }=28.3^{\circ}, \theta_{\min }=1.8^{\circ}$

$h=-8 \rightarrow 8$

$k=-13 \rightarrow 13$

$l=-15 \rightarrow 15$

Secondary atom site location: difference Fourier map

Hydrogen site location: difference Fourier map

$\mathrm{H}$-atom parameters constrained

$w=1 /\left[\sigma^{2}\left(F_{\mathrm{o}}^{2}\right)+(0.0431 P)^{2}+0.3244 P\right]$

where $P=\left(F_{\mathrm{o}}^{2}+2 F_{\mathrm{c}}{ }^{2}\right) / 3$

$(\Delta / \sigma)_{\max }=0.001$

$\Delta \rho_{\max }=0.57 \mathrm{e} \AA^{-3}$

$\Delta \rho_{\min }=-0.59$ e $\AA^{-3}$

Special details

Geometry. All esds (except the esd in the dihedral angle between two 1.s. planes) are estimated using the full covariance matrix. The cell esds are taken into account individually in the estimation of esds in distances, angles and torsion angles; correlations between esds in cell parameters are only used when they are defined by crystal symmetry. An approximate (isotropic) treatment of cell esds is used for estimating esds involving 1.s. planes.

Refinement. Refinement of $\mathrm{F}^{2}$ against ALL reflections. The weighted R-factor $\mathrm{wR}$ and goodness of fit $\mathrm{S}$ are based on $\mathrm{F}^{2}$, conventional R-factors $R$ are based on $F$, with $F$ set to zero for negative $F^{2}$. The threshold expression of $F^{2}>2 \operatorname{sigma}\left(F^{2}\right)$ is used only for calculating R-factors(gt) etc. and is not relevant to the choice of reflections for refinement. R-factors based on $\mathrm{F}^{2}$ are statistically about twice as large as those based on F, and R- factors based on ALL data will be even larger.

Fractional atomic coordinates and isotropic or equivalent isotropic displacement parameters $\left(\AA^{2}\right)$

\begin{tabular}{lllll}
\hline & $x$ & $y$ & $z$ & $U_{\text {iso }} * / U_{\text {eq }}$ \\
\hline Br1 & $1.23629(4)$ & $0.91785(2)$ & $0.915580(19)$ & $0.04170(10)$ \\
S1 & $0.69071(9)$ & $0.27380(6)$ & $0.84528(4)$ & $0.03194(13)$ \\
O1 & $0.8013(2)$ & $0.10195(16)$ & $0.53135(13)$ & $0.0336(3)$
\end{tabular}




$\begin{array}{lllll}\text { O2 } & 0.4646(3) & 0.2757(2) & 0.86938(15) & 0.0462(4) \\ \text { C1 } & 0.6970(3) & 0.2139(2) & 0.69530(17) & 0.0274(4) \\ \text { C2 } & 0.5786(3) & 0.2405(2) & 0.59942(17) & 0.0265(4) \\ \text { C3 } & 0.4234(4) & 0.3139(2) & 0.5861(2) & 0.0332(5) \\ \text { H3 } & 0.3725 & 0.3633 & 0.6524 & 0.040^{*} \\ \text { C4 } & 0.3442(4) & 0.3134(3) & 0.4742(2) & 0.0396(5) \\ \text { C5 } & 0.4239(4) & 0.2411(3) & 0.3781(2) & 0.0453(6) \\ \text { H5 } & 0.3709 & 0.2433 & 0.3022 & 0.054^{*} \\ \text { C6 } & 0.5762(4) & 0.1665(3) & 0.3889(2) & 0.0410(5) \\ \text { H6 } & 0.6276 & 0.1169 & 0.3229 & 0.049^{*} \\ \text { C7 } & 0.6492(3) & 0.1686(2) & 0.50126(18) & 0.0303(4) \\ \text { C8 } & 0.8253(3) & 0.1306(2) & 0.65040(18) & 0.0298(4) \\ \text { C9 } & 0.1740(4) & 0.3903(3) & 0.4573(3) & 0.0570(8) \\ \text { H9A } & 0.0339 & 0.3220 & 0.4338 & 0.086^{*} \\ \text { H9B } & 0.2114 & 0.4437 & 0.3966 & 0.086^{*} \\ \text { H9C } & 0.1660 & 0.4550 & 0.5305 & 0.086^{*} \\ \text { C10 } & 0.9846(4) & 0.0710(3) & 0.7040(2) & 0.0406(5) \\ \text { H10A } & 1.1308 & 0.1223 & 0.6938 & 0.061^{*} \\ \text { H10B } & 0.9612 & -0.0286 & 0.6664 & 0.061^{*} \\ \text { H10C } & 0.9671 & 0.0797 & 0.7874 & 0.061^{*} \\ \text { C11 } & 0.8359(3) & 0.4538(2) & 0.85581(16) & 0.0277(4) \\ \text { C12 } & 0.7417(4) & 0.5610(2) & 0.89976(19) & 0.0334(5) \\ \text { H12 } & 0.5963 & 0.5394 & 0.9183 & 0.040^{*} \\ \text { C13 } & 0.8589(4) & 0.7004(2) & 0.91705(19) & 0.0354(5) \\ \text { H13 } & 0.7951 & 0.7747 & 0.9475 & 0.042^{*} \\ \text { C14 } & 1.0693(4) & 0.7290(2) & 0.88928(17) & 0.0304(4) \\ \text { C15 } & 1.1664(4) & 0.6220(2) & 0.84572(19) & 0.0344(5) \\ \text { H15 } & 1.3117 & 0.6438 & 0.8269 & 0.041^{*} \\ \text { C16 } & 1.0498(4) & 0.4837(2) & 0.83008(19) & 0.0342(5) \\ \text { H16 } & 1.1152 & 0.4094 & 0.8019 & 0.041^{*}\end{array}$

Atomic displacement parameters $\left(\AA^{2}\right)$

\begin{tabular}{lllllll}
\hline & $U^{11}$ & $U^{22}$ & $U^{33}$ & $U^{12}$ & $U^{13}$ & $U^{23}$ \\
\hline Br1 & $0.05053(18)$ & $0.03378(14)$ & $0.03637(14)$ & $0.00588(11)$ & $0.00285(10)$ & $0.00355(9)$ \\
S1 & $0.0324(3)$ & $0.0376(3)$ & $0.0233(2)$ & $0.0047(2)$ & $0.0038(2)$ & $0.0056(2)$ \\
O1 & $0.0309(8)$ & $0.0348(8)$ & $0.0329(8)$ & $0.0088(7)$ & $0.0073(6)$ & $0.0011(6)$ \\
O2 & $0.0323(9)$ & $0.0570(11)$ & $0.0400(9)$ & $-0.0004(8)$ & $0.0154(7)$ & $0.0009(8)$ \\
C1 & $0.0257(10)$ & $0.0285(10)$ & $0.0262(9)$ & $0.0053(8)$ & $0.0017(8)$ & $0.0039(8)$ \\
C2 & $0.0227(10)$ & $0.0271(10)$ & $0.0267(9)$ & $0.0010(8)$ & $0.0024(7)$ & $0.0051(8)$ \\
C3 & $0.0261(11)$ & $0.0345(11)$ & $0.0393(12)$ & $0.0057(9)$ & $0.0036(9)$ & $0.0107(9)$ \\
C4 & $0.0250(11)$ & $0.0403(12)$ & $0.0530(14)$ & $-0.0023(9)$ & $-0.0041(10)$ & $0.0235(11)$ \\
C5 & $0.0367(13)$ & $0.0582(16)$ & $0.0352(12)$ & $-0.0070(11)$ & $-0.0066(10)$ & $0.0214(11)$ \\
C6 & $0.0392(13)$ & $0.0504(14)$ & $0.0256(10)$ & $-0.0011(11)$ & $0.0024(9)$ & $0.0056(9)$ \\
C7 & $0.0263(11)$ & $0.0316(10)$ & $0.0284(10)$ & $0.0005(8)$ & $0.0028(8)$ & $0.0042(8)$ \\
C8 & $0.0266(11)$ & $0.0262(10)$ & $0.0334(10)$ & $0.0036(8)$ & $0.0022(8)$ & $0.0032(8)$ \\
C9 & $0.0308(14)$ & $0.0602(17)$ & $0.086(2)$ & $0.0058(12)$ & $-0.0083(13)$ & $0.0402(16)$ \\
C10 & $0.0327(13)$ & $0.0368(12)$ & $0.0536(14)$ & $0.0123(10)$ & $0.0005(11)$ & $0.0092(10)$
\end{tabular}


supporting information

\begin{tabular}{lllllll}
$\mathrm{C} 11$ & $0.0273(10)$ & $0.0342(10)$ & $0.0201(9)$ & $0.0073(8)$ & $0.0007(7)$ & $0.0029(7)$ \\
$\mathrm{C} 12$ & $0.0263(11)$ & $0.0439(12)$ & $0.0320(11)$ & $0.0135(9)$ & $0.0066(8)$ & $0.0065(9)$ \\
$\mathrm{C} 13$ & $0.0388(13)$ & $0.0388(12)$ & $0.0316(10)$ & $0.0182(10)$ & $0.0056(9)$ & $0.0038(9)$ \\
$\mathrm{C} 14$ & $0.0350(12)$ & $0.0308(10)$ & $0.0224(9)$ & $0.0061(9)$ & $-0.0023(8)$ & $0.0026(8)$ \\
$\mathrm{C} 15$ & $0.0263(11)$ & $0.0395(12)$ & $0.0358(11)$ & $0.0090(9)$ & $0.0052(9)$ & $0.0035(9)$ \\
$\mathrm{C} 16$ & $0.0303(11)$ & $0.0372(12)$ & $0.0344(11)$ & $0.0117(9)$ & $0.0079(9)$ & $0.0011(9)$ \\
\hline
\end{tabular}

Geometric parameters $\left(\AA,{ }^{o}\right)$

\begin{tabular}{|c|c|c|c|}
\hline $\mathrm{Br} 1-\mathrm{C} 14$ & $1.895(2)$ & $\mathrm{C} 8-\mathrm{C} 10$ & $1.482(3)$ \\
\hline $\mathrm{S} 1-\mathrm{O} 2$ & $1.4927(18)$ & C9-H9A & 0.9800 \\
\hline $\mathrm{S} 1-\mathrm{C} 1$ & $1.751(2)$ & C9-H9B & 0.9800 \\
\hline $\mathrm{S} 1-\mathrm{C} 11$ & $1.798(2)$ & $\mathrm{C} 9-\mathrm{H} 9 \mathrm{C}$ & 0.9800 \\
\hline $\mathrm{O} 1-\mathrm{C} 8$ & $1.367(3)$ & $\mathrm{C} 10-\mathrm{H} 10 \mathrm{~A}$ & 0.9800 \\
\hline $\mathrm{O} 1-\mathrm{C} 7$ & $1.381(3)$ & $\mathrm{C} 10-\mathrm{H} 10 \mathrm{~B}$ & 0.9800 \\
\hline $\mathrm{C} 1-\mathrm{C} 8$ & $1.357(3)$ & $\mathrm{C} 10-\mathrm{H} 10 \mathrm{C}$ & 0.9800 \\
\hline $\mathrm{C} 1-\mathrm{C} 2$ & $1.439(3)$ & $\mathrm{C} 11-\mathrm{C} 12$ & $1.379(3)$ \\
\hline $\mathrm{C} 2-\mathrm{C} 7$ & $1.386(3)$ & $\mathrm{C} 11-\mathrm{C} 16$ & $1.391(3)$ \\
\hline $\mathrm{C} 2-\mathrm{C} 3$ & $1.395(3)$ & $\mathrm{C} 12-\mathrm{C} 13$ & $1.388(3)$ \\
\hline $\mathrm{C} 3-\mathrm{C} 4$ & $1.391(3)$ & $\mathrm{C} 12-\mathrm{H} 12$ & 0.9500 \\
\hline $\mathrm{C} 3-\mathrm{H} 3$ & 0.9500 & $\mathrm{C} 13-\mathrm{C} 14$ & $1.377(3)$ \\
\hline $\mathrm{C} 4-\mathrm{C} 5$ & $1.399(4)$ & $\mathrm{C} 13-\mathrm{H} 13$ & 0.9500 \\
\hline $\mathrm{C} 4-\mathrm{C} 9$ & $1.508(3)$ & $\mathrm{C} 14-\mathrm{C} 15$ & $1.389(3)$ \\
\hline $\mathrm{C} 5-\mathrm{C} 6$ & $1.382(4)$ & $\mathrm{C} 15-\mathrm{C} 16$ & $1.379(3)$ \\
\hline $\mathrm{C} 5-\mathrm{H} 5$ & 0.9500 & $\mathrm{C} 15-\mathrm{H} 15$ & 0.9500 \\
\hline $\mathrm{C} 6-\mathrm{C} 7$ & $1.380(3)$ & $\mathrm{C} 16-\mathrm{H} 16$ & 0.9500 \\
\hline $\mathrm{C} 6-\mathrm{H} 6$ & 0.9500 & & \\
\hline $\mathrm{O} 2-\mathrm{S} 1-\mathrm{C} 1$ & $108.74(10)$ & $\mathrm{C} 4-\mathrm{C} 9-\mathrm{H} 9 \mathrm{~B}$ & 109.5 \\
\hline $\mathrm{O} 2-\mathrm{S} 1-\mathrm{C} 11$ & $106.52(10)$ & H9A-C9-H9B & 109.5 \\
\hline $\mathrm{C} 1-\mathrm{S} 1-\mathrm{C} 11$ & $97.81(10)$ & $\mathrm{C} 4-\mathrm{C} 9-\mathrm{H} 9 \mathrm{C}$ & 109.5 \\
\hline $\mathrm{C} 8-\mathrm{O} 1-\mathrm{C} 7$ & $106.09(16)$ & $\mathrm{H} 9 \mathrm{~A}-\mathrm{C} 9-\mathrm{H} 9 \mathrm{C}$ & 109.5 \\
\hline $\mathrm{C} 8-\mathrm{C} 1-\mathrm{C} 2$ & $107.82(18)$ & $\mathrm{H} 9 \mathrm{~B}-\mathrm{C} 9-\mathrm{H} 9 \mathrm{C}$ & 109.5 \\
\hline $\mathrm{C} 8-\mathrm{C} 1-\mathrm{S} 1$ & $122.92(16)$ & $\mathrm{C} 8-\mathrm{C} 10-\mathrm{H} 10 \mathrm{~A}$ & 109.5 \\
\hline $\mathrm{C} 2-\mathrm{C} 1-\mathrm{S} 1$ & $129.26(16)$ & $\mathrm{C} 8-\mathrm{C} 10-\mathrm{H} 10 \mathrm{~B}$ & 109.5 \\
\hline $\mathrm{C} 7-\mathrm{C} 2-\mathrm{C} 3$ & $119.4(2)$ & $\mathrm{H} 10 \mathrm{~A}-\mathrm{C} 10-\mathrm{H} 10 \mathrm{~B}$ & 109.5 \\
\hline $\mathrm{C} 7-\mathrm{C} 2-\mathrm{C} 1$ & $104.22(18)$ & $\mathrm{C} 8-\mathrm{C} 10-\mathrm{H} 10 \mathrm{C}$ & 109.5 \\
\hline $\mathrm{C} 3-\mathrm{C} 2-\mathrm{C} 1$ & $136.4(2)$ & $\mathrm{H} 10 \mathrm{~A}-\mathrm{C} 10-\mathrm{H} 10 \mathrm{C}$ & 109.5 \\
\hline $\mathrm{C} 4-\mathrm{C} 3-\mathrm{C} 2$ & $118.9(2)$ & $\mathrm{H} 10 \mathrm{~B}-\mathrm{C} 10-\mathrm{H} 10 \mathrm{C}$ & 109.5 \\
\hline $\mathrm{C} 4-\mathrm{C} 3-\mathrm{H} 3$ & 120.6 & $\mathrm{C} 12-\mathrm{C} 11-\mathrm{C} 16$ & $120.6(2)$ \\
\hline $\mathrm{C} 2-\mathrm{C} 3-\mathrm{H} 3$ & 120.6 & $\mathrm{C} 12-\mathrm{C} 11-\mathrm{S} 1$ & $119.81(17)$ \\
\hline $\mathrm{C} 3-\mathrm{C} 4-\mathrm{C} 5$ & $119.4(2)$ & $\mathrm{C} 16-\mathrm{C} 11-\mathrm{S} 1$ & $119.33(16)$ \\
\hline $\mathrm{C} 3-\mathrm{C} 4-\mathrm{C} 9$ & $120.0(3)$ & $\mathrm{C} 11-\mathrm{C} 12-\mathrm{C} 13$ & $120.2(2)$ \\
\hline $\mathrm{C} 5-\mathrm{C} 4-\mathrm{C} 9$ & $120.6(2)$ & $\mathrm{C} 11-\mathrm{C} 12-\mathrm{H} 12$ & 119.9 \\
\hline $\mathrm{C} 6-\mathrm{C} 5-\mathrm{C} 4$ & $122.9(2)$ & $\mathrm{C} 13-\mathrm{C} 12-\mathrm{H} 12$ & 119.9 \\
\hline $\mathrm{C} 6-\mathrm{C} 5-\mathrm{H} 5$ & 118.5 & $\mathrm{C} 14-\mathrm{C} 13-\mathrm{C} 12$ & $118.8(2)$ \\
\hline $\mathrm{C} 4-\mathrm{C} 5-\mathrm{H} 5$ & 118.5 & $\mathrm{C} 14-\mathrm{C} 13-\mathrm{H} 13$ & 120.6 \\
\hline $\mathrm{C} 7-\mathrm{C} 6-\mathrm{C} 5$ & $115.9(2)$ & $\mathrm{C} 12-\mathrm{C} 13-\mathrm{H} 13$ & 120.6 \\
\hline
\end{tabular}




\begin{tabular}{|c|c|c|c|}
\hline $\mathrm{C} 7-\mathrm{C} 6-\mathrm{H} 6$ & 122.0 & $\mathrm{C} 13-\mathrm{C} 14-\mathrm{C} 15$ & $121.5(2)$ \\
\hline $\mathrm{C} 5-\mathrm{C} 6-\mathrm{H} 6$ & 122.0 & $\mathrm{C} 13-\mathrm{C} 14-\mathrm{Br} 1$ & $119.97(16)$ \\
\hline $\mathrm{C} 6-\mathrm{C} 7-\mathrm{O} 1$ & $125.3(2)$ & $\mathrm{C} 15-\mathrm{C} 14-\mathrm{Br} 1$ & $118.46(17)$ \\
\hline $\mathrm{C} 6-\mathrm{C} 7-\mathrm{C} 2$ & $123.5(2)$ & $\mathrm{C} 16-\mathrm{C} 15-\mathrm{C} 14$ & $119.3(2)$ \\
\hline $\mathrm{O} 1-\mathrm{C} 7-\mathrm{C} 2$ & $111.16(18)$ & $\mathrm{C} 16-\mathrm{C} 15-\mathrm{H} 15$ & 120.3 \\
\hline $\mathrm{C} 1-\mathrm{C} 8-\mathrm{O} 1$ & $110.71(18)$ & $\mathrm{C} 14-\mathrm{C} 15-\mathrm{H} 15$ & 120.3 \\
\hline $\mathrm{C} 1-\mathrm{C} 8-\mathrm{C} 10$ & $133.1(2)$ & $\mathrm{C} 15-\mathrm{C} 16-\mathrm{C} 11$ & $119.6(2)$ \\
\hline $\mathrm{O} 1-\mathrm{C} 8-\mathrm{C} 10$ & $116.15(19)$ & $\mathrm{C} 15-\mathrm{C} 16-\mathrm{H} 16$ & 120.2 \\
\hline $\mathrm{C} 4-\mathrm{C} 9-\mathrm{H} 9 \mathrm{~A}$ & 109.5 & $\mathrm{C} 11-\mathrm{C} 16-\mathrm{H} 16$ & 120.2 \\
\hline $\mathrm{O} 2-\mathrm{S} 1-\mathrm{C} 1-\mathrm{C} 8$ & $-143.25(19)$ & $\mathrm{C} 1-\mathrm{C} 2-\mathrm{C} 7-\mathrm{O} 1$ & $-0.3(2)$ \\
\hline $\mathrm{C} 11-\mathrm{S} 1-\mathrm{C} 1-\mathrm{C} 8$ & $106.29(19)$ & $\mathrm{C} 2-\mathrm{C} 1-\mathrm{C} 8-\mathrm{O} 1$ & $0.8(2)$ \\
\hline $\mathrm{O} 2-\mathrm{S} 1-\mathrm{C} 1-\mathrm{C} 2$ & $37.3(2)$ & $\mathrm{S} 1-\mathrm{C} 1-\mathrm{C} 8-\mathrm{O} 1$ & $-178.83(14)$ \\
\hline $\mathrm{C} 11-\mathrm{S} 1-\mathrm{C} 1-\mathrm{C} 2$ & $-73.2(2)$ & $\mathrm{C} 2-\mathrm{C} 1-\mathrm{C} 8-\mathrm{C} 10$ & $178.7(2)$ \\
\hline $\mathrm{C} 8-\mathrm{C} 1-\mathrm{C} 2-\mathrm{C} 7$ & $-0.3(2)$ & $\mathrm{S} 1-\mathrm{C} 1-\mathrm{C} 8-\mathrm{C} 10$ & $-0.9(4)$ \\
\hline $\mathrm{S} 1-\mathrm{C} 1-\mathrm{C} 2-\mathrm{C} 7$ & $179.30(17)$ & $\mathrm{C} 7-\mathrm{O} 1-\mathrm{C} 8-\mathrm{C} 1$ & $-1.0(2)$ \\
\hline $\mathrm{C} 8-\mathrm{C} 1-\mathrm{C} 2-\mathrm{C} 3$ & $178.9(2)$ & $\mathrm{C} 7-\mathrm{O} 1-\mathrm{C} 8-\mathrm{C} 10$ & $-179.27(19)$ \\
\hline $\mathrm{S} 1-\mathrm{C} 1-\mathrm{C} 2-\mathrm{C} 3$ & $-1.5(4)$ & $\mathrm{O} 2-\mathrm{S} 1-\mathrm{C} 11-\mathrm{C} 12$ & $10.2(2)$ \\
\hline $\mathrm{C} 7-\mathrm{C} 2-\mathrm{C} 3-\mathrm{C} 4$ & $-0.3(3)$ & $\mathrm{C} 1-\mathrm{S} 1-\mathrm{C} 11-\mathrm{C} 12$ & $122.51(17)$ \\
\hline $\mathrm{C} 1-\mathrm{C} 2-\mathrm{C} 3-\mathrm{C} 4$ & $-179.4(2)$ & $\mathrm{O} 2-\mathrm{S} 1-\mathrm{C} 11-\mathrm{C} 16$ & $-175.97(17)$ \\
\hline $\mathrm{C} 2-\mathrm{C} 3-\mathrm{C} 4-\mathrm{C} 5$ & $-0.8(3)$ & $\mathrm{C} 1-\mathrm{S} 1-\mathrm{C} 11-\mathrm{C} 16$ & $-63.70(18)$ \\
\hline $\mathrm{C} 2-\mathrm{C} 3-\mathrm{C} 4-\mathrm{C} 9$ & $179.1(2)$ & $\mathrm{C} 16-\mathrm{C} 11-\mathrm{C} 12-\mathrm{C} 13$ & $1.1(3)$ \\
\hline $\mathrm{C} 3-\mathrm{C} 4-\mathrm{C} 5-\mathrm{C} 6$ & $1.4(4)$ & $\mathrm{S} 1-\mathrm{C} 11-\mathrm{C} 12-\mathrm{C} 13$ & $174.76(16)$ \\
\hline $\mathrm{C} 9-\mathrm{C} 4-\mathrm{C} 5-\mathrm{C} 6$ & $-178.5(2)$ & $\mathrm{C} 11-\mathrm{C} 12-\mathrm{C} 13-\mathrm{C} 14$ & $0.2(3)$ \\
\hline $\mathrm{C} 4-\mathrm{C} 5-\mathrm{C} 6-\mathrm{C} 7$ & $-0.8(4)$ & $\mathrm{C} 12-\mathrm{C} 13-\mathrm{C} 14-\mathrm{C} 15$ & $-0.6(3)$ \\
\hline $\mathrm{C} 5-\mathrm{C} 6-\mathrm{C} 7-\mathrm{O} 1$ & $-179.6(2)$ & $\mathrm{C} 12-\mathrm{C} 13-\mathrm{C} 14-\mathrm{Br} 1$ & $-178.34(16)$ \\
\hline $\mathrm{C} 5-\mathrm{C} 6-\mathrm{C} 7-\mathrm{C} 2$ & $-0.4(4)$ & $\mathrm{C} 13-\mathrm{C} 14-\mathrm{C} 15-\mathrm{C} 16$ & $-0.1(3)$ \\
\hline $\mathrm{C} 8-\mathrm{O} 1-\mathrm{C} 7-\mathrm{C} 6$ & $-179.9(2)$ & $\mathrm{Br} 1-\mathrm{C} 14-\mathrm{C} 15-\mathrm{C} 16$ & $177.61(17)$ \\
\hline $\mathrm{C} 8-\mathrm{O} 1-\mathrm{C} 7-\mathrm{C} 2$ & $0.8(2)$ & $\mathrm{C} 14-\mathrm{C} 15-\mathrm{C} 16-\mathrm{C} 11$ & $1.3(3)$ \\
\hline $\mathrm{C} 3-\mathrm{C} 2-\mathrm{C} 7-\mathrm{C} 6$ & $0.9(3)$ & $\mathrm{C} 12-\mathrm{C} 11-\mathrm{C} 16-\mathrm{C} 15$ & $-1.8(3)$ \\
\hline $\mathrm{C} 1-\mathrm{C} 2-\mathrm{C} 7-\mathrm{C} 6$ & $-179.7(2)$ & $\mathrm{S} 1-\mathrm{C} 11-\mathrm{C} 16-\mathrm{C} 15$ & $-175.56(17)$ \\
\hline $\mathrm{C} 3-\mathrm{C} 2-\mathrm{C} 7-\mathrm{O} 1$ & $-179.69(18)$ & & \\
\hline
\end{tabular}

